\title{
Monofilamento: Conhecimento sobre sua Utilização
}

\author{
Monofilament: Knowledge about their Use
}

Monofilamento: Conocimientos sobre su Uso

Cristiane Costa Reis Silva', Najhara Shanna Santiago Souza', Tamilis Fernanda Moreira Souza ${ }^{1}$

\begin{abstract}
RESUMO
O monofilamento Semmes-Weinstein é um teste de sensibilidade realizado em pacientes acometidos por neuropatia periférica decorrente de diabetes mellitus e hanseníase, quando não há diagnóstico precoce. O presente trabalho teve como objetivo geral identificar o conhecimento sobre o referido teste por parte de discentes de Enfermagem de uma instituição de ensino superior privada situada no município de Salvador, Estado da Bahia. Trata-se de pesquisa de campo com estudo descritivo quantitativo por meio da aplicação de questionário composto de perguntas objetivas e subjetivas. A opção das pesquisadoras pelo tema justifica-se pela carência de estudos sobre o teste durante a graduação e pela importância de ampliar o conhecimento a respeito desse instrumento de avaliação que auxilia no diagnóstico precoce da doença, evitando, assim, lesões nos nervos e eventual amputação de membros. Com base nos resultados obtidos, observa-se que os discentes de Enfermagem têm concluído a graduação sem dispor de orientação sobre a importância e a utilização do monofilamento de Semmes-Weinstein.
\end{abstract}

DESCRITORES: Sistema nervoso periférico. Conhecimento. Enfermagem. Estomaterapia.

\begin{abstract}
The Semmes-Weinstein monofilament is a sensitivity test performed in patients with peripheral neuropathy caused by diabetes mellitus and leprosy, when there is early diagnosis. The present work aimed at identifying the general knowledge about the aforementioned test by Nursing students of a private institution of higher learning in the municipality of Salvador, Bahia State. This is field research with quantitative descriptive study through the application of a questionnaire composed of objective and subjective questions. The option of researchers by theme is justified by the lack of studies on the test for graduation and the importance of increasing the knowledge about this assessment tool that assists in the early diagnosis of the disease, thus avoiding nerve damage and possible amputation of members. Based on the results obtained, it is observed that the Nursing students have completed graduation without guidance on the importance and use of the Semmes-Weinstein monofilament.
\end{abstract}

DESCRIPTORS: Peripheral nervous system. Knowledge. Nursing. Stomatherapy.

\section{RESUMEN}

El monofilamento de Semmes-Weinstein es un test de sensibilidad realizada en pacientes con neuropatía periférica resultante de diabetes mellitus y lepra, cuando no hay diagnóstico precoz. El presente trabajo tuvo como objetivo general identificar el conocimiento sobre el referido test por parte estudiantes de Enfermería de una institución privada de educación superior situada en el municipio de Salvador, estado de Bahía. Se trata de investigación de campo con estudio descriptivo cuantitativo mediante la aplicación de un cuestionario compuesto de preguntas subjetivas y objetivas. La opción de los investigadores por el tema se justifica por la carencia de estudios sobre el test durante la graduación y por la importancia de ampliar el conocimiento respecto a este instrumento de evaluación que ayuda en el diagnóstico precoz de la enfermedad, evitando así lesiones en los nervios y eventual amputación de miembros. Basado en los resultados obtenidos, se observa que los estudiantes de Enfermería han terminado la graduación sin disponer de orientación sobre la importancia y el uso del monofilamento de Semmes-Weinstein.

DESCRIPTORES: Sistema nervioso periférico. Conocimiento. Enfermería. Estomaterapia.

\footnotetext{
${ }^{1}$ Universidade Salvador (UNIFACS) - Salvador (BA), Brasil.

Endereço para correspondência: Rua Bombeiro Eliezer de Alexandrino, 107 - Condomínio Residencial dos Coqueiros, Edifício Itacimirin, Apto. 203 CEP: 41710-790 - Salvador (BA), Brasil - E-mail: najhara.santiago@hotmail.com

Artigo recebido em: 20/07/2015 - Aceito para publicação em: 13/06/2016
} 


\section{INTRODUÇÃO}

Os nervos periféricos do corpo humano são compostos por três elementos - sensoriais, motores e autônomos. A neuropatia periférica ocorre quando algum deles é atingido de forma isolada ou simultaneamente ${ }^{1}$. Atualmente, estima-se que cerca de $2 \%$ da população em geral apresentem neuropatia periférica; quando considerados adultos com mais de 55 anos, esse valor sobe para $8 \%^{2}$.

A doença pode ser classificada em três tipos: mononeuropatia, que atinge apenas um tronco nervoso; mononeuropatias múltiplas, quando vários troncos nervosos são atingidos; e polineuropatia distal, que acomete os quatro membros de forma difusa e simétrica. A neuropatia periférica pode ocorrer em pacientes que apresentam doenças diversas, entre elas diabetes mellitus e hanseníase, nos quais são comuns alterações sensitivas e autonômicas ${ }^{2}$.

Dados epidemiológicos demonstram ser crescente o número de indivíduos com diabetes mellitus e hanseníase. No ano de 2015, segundo dados do Sistema de Informação de Agravos de Notificação (SINAN), foram confirmados e notificados 3.025 casos de hanseníase na Bahia ${ }^{3}$. Somente em Salvador foram 488 casos, seguida da cidade de Araci, com 196. Levantamento do Departamento de Informática do Sistema Único de Saúde (DATASUS), por meio do Sistema de Cadastramento e Acompanhamento de Hipertensos e Diabéticos no município de Salvador (BA), revela que nos meses de janeiro a abril de 2013 houve a identificação de 347 pacientes com diabetes mellitus, sendo 101 deles do diabetes tipo 1 e 246 do tipo $2^{4}$.

Para evitar a progressão da neuropatia periférica, sem consequências para o indivíduo, este deverá ser acompanhado por um médico que irá realizar a anamnese, encaminhá-lo para exames de controle glicêmico e indicar testes que avaliarão a sensibilidade periférica. Essa avaliação tem papel fundamental no diagnóstico e tratamento de lesões dos nervos periféricos, pois a detecção precoce de distúrbios da função nervosa é de extrema importância, visto que a intervenção médica e da equipe multidisciplinar pode evitar a perda progressiva e permanente da funcionalidade do nervo afetado, além do risco de ulcerações e incapacidade de mobilidade do indivíduo 5 .

Um dos diagnósticos para a neuropatia é realizado por meio do teste de sensibilidade com monofilamento de Semmes-Weinstein de gramas variados, cujo objetivo consiste em avaliar a sensibilidade tátil dos pacientes com possível lesão nos nervos. O instrumento de avaliação é feito com um estesiômetro de náilon, o qual é aplicado suavemente em cerca de cinco pontos de pressão nos pés e/ou mãos do paciente . $^{6}$

O uso desses monofilamentos para detecção de presença/ ausência de sensibilidade teve início no ano de 1800. Foram desenvolvidos pelo neurofisiologista Josephine Semmes e por Sidney Weinstein, neuropsicólogo. Realizou-se o teste pela primeira vez com pelos de cavalos e, posteriormente, já no ano de 1960, utilizou-se o atual monofilamento de náilon ${ }^{7}$.

Tempo depois, em 1983, a Coordenação Nacional de Dermatologia Sanitária do Ministério da Saúde do Brasil recomendou que fosse implantada a utilização dos monofilamentos de Semmes-Weinstein para avaliação dos nervos periféricos, com as outras ações previstas no Programa de Controle da Hanseníase ${ }^{8}$. Acresce-se estar incluída no Protocolo de Atendimento de Hipertensão e Diabetes da Prefeitura de São Paulo (2011) a orientação para que pacientes com diabetes mellitus sejam submetidos a uma rotina de procedimentos na primeira consulta e realizem uma avaliação anual, durante a qual serão feitos exames laboratoriais, aferidas as medidas antropométricas, além do exame de sensibilidade com monofilamento de Semmes-Weinstein, cujo intuito é identificar aqueles que apresentam risco de ulceração?

Além disso, na consulta de enfermagem, os profissionais devem coletar as informações necessárias, transmiti-las à equipe médica e multiprofissional, a fim de estabelecer um diagnóstico preciso e fornecer orientações aos pacientes e familiares sobre a importância do autocuidado. Quando se trata de neuropatia periférica, é fundamental também que os profissionais conheçam o teste de sensibilidade com monofilamentos de Semmes-Weinstein e o benefício que ele proporciona ao paciente, ou seja, o diagnóstico precoce que reduz a chance de lesões nos nervos. Devem também dispor de informações sobre a sua utilização e aplicação ${ }^{10}$.

Diante do exposto, este estudo assume grande relevância prática, por tratar de um teste atualmente disponível e utilizado no Programa de Saúde da Família (PSF), de baixo custo, fácil manuseio e, ainda, por haver grande número de pessoas acometidas com doenças que alteram a sensibilidade e, portanto, possivelmente beneficiadas com a sua maior utilização. Para tanto, são necessários profissionais de saúde capacitados a realizar essa avaliação.

O desenvolvimento do presente estudo justificou-se também pelo pouco contato dos alunos de Enfermagem com esse instrumento de avaliação, o que despertou o interesse das autoras em melhor investigar o conhecimento a seu respeito. 


\section{OBJETIVOS}

Este estudo teve como objetivo geral identificar o conhecimento de discentes de Enfermagem da instituição campo de estudo sobre monofilamento de Semmes-Weinstein e, como objetivos específicos, identificar quantos desses discentes conhecem a técnica de aplicação do monofilamento de Semmes-Weinstein e verificar a dificuldade por eles encontrada para aplicá-lo.

\section{MÉTODOS}

Trata-se de pesquisa de campo, com estudo descritivo e abordagem quantitativa.Participaram 26 discentes de Enfermagem de uma instituição de ensino superior privada, localizada no município de Salvador, Estado Bahia, no ano de 2015.

Os participantes cursavam o nono e o décimo semestres de curso. Constituíram critérios de exclusão na pesquisa: a não assinatura do Termo de Consentimento Livre e Esclarecido, e o trancamento de matrícula no curso.

Optou-se por selecionar estudantes que cursassem os dois referidos semestres em virtude de apenas três estarem no décimo período na instituição. Dessa forma, incluir discentes também do nono semestre possibilitou a coleta das informações necessárias com um maior número de participantes.

A pesquisa foi realizada após obtenção do parecer favorável ( $n^{\circ}$ 919.778) do Comitê de Ética em Pesquisa da própria Universidade, via Plataforma Brasil. As pesquisadoras coletaram os dados no mês de março de 2015, em uma sala de aula, momento em que o projeto de pesquisa pôde ser apresentado aos participantes, bem como foram feitas orientações sobre o questionário e realizada a assinatura do Termo de Consentimento Livre e Esclarecido após sua concordância, conforme preconizado na Resolução do Conselho Nacional de Saúde no 466/12.

Aos participantes foi entregue um questionário composto de 15 questões objetivas e subjetivas sobre o tema investigado, o qual foi recolhido logo após eles serem informados sobre o objetivo do estudo e assegurados quanto ao sigilo das informações.

Após a coleta dos dados por meio da aplicação do questionário, realizou-se a tabulação no programa do Excel. Optou-se por organizar e apresentar os dados em gráficos e tabelas, com cálculo de porcentagem das seguintes variáveis: características sociodemográficas e específicas, como sexo e idade; informação sobre monofilamento; importância da utilização dos monofilamentos; vantagens; contraindicação; onde se obteve conhecimento sobre os monofilamentos; qual é o profissional habilitado a realizar o exame de sensibilidade; e se durante o período curricular houve contato com esse instrumento de avaliação.

\section{RESULTADOS E DISCUSSÃO}

A análise das características sociodemográficas dos participantes mostra que 25 deles são do sexo feminino, e 1, do masculino. Já quando observada a idade, 21 têm entre $21 \mathrm{e}$ 30 anos; 2, entre 31 e 40 anos; 2, 20 anos ou menos; e 1 não informou a idade (Gráficos 1 e 2 e Tabela 1).

Conforme dados coletados na pesquisa, $92,3 \%$ dos participantes relataram desconhecer o monofilamento de Semmes-Weinstein, e apenas 7,7\% afirmaram ter informações a respeito. Diante desse achado, é importante pontuar que a aquisição de conhecimento e experiência prática durante a formação profissional é de suma importância para o desenvolvimento de habilidades, além de qualificar o profissional e possibilitar o seu aprimoramento.

Entretanto, apesar de ser um conhecimento importante a esses futuros profissionais, $69 \%$ dos discentes que participaram deste estudo informaram desconhecer ou não julgar importante a utilização do monofilamento de SemmesWeinstein, apenas 8,0\% afirmaram reconhecer a importância do teste, e $23 \%$ não responderam.

Mesmo entre aqueles que afirmaram ter informações houve equívocos, pois relataram considerar o referido teste importante para rastrear pacientes, a fim de evitar agravos de lesões, como o pé diabético. Isso evidencia que os discentes de Enfermagem estão concluindo a graduação sem conhecer o teste de sensibilidade com monofilamento de Semmes-Weinstein, apesar de constituir instrumento de avaliação atualmente disponível na rede pública e bastante utilizado em programas de atenção à saúde de pacientes com diabetes e hanseníase, com o intuito de avaliar a sensibilidade desses pacientes.

O estesiômetro foi desenvolvido por Von Frey utilizando fios de cabelo e pelos de cavalo, em 1800, com o objetivo de analisar a pressão e o tato. Posteriormente, Semmes e Weinstein começaram a utilizar os monofilamentos de 


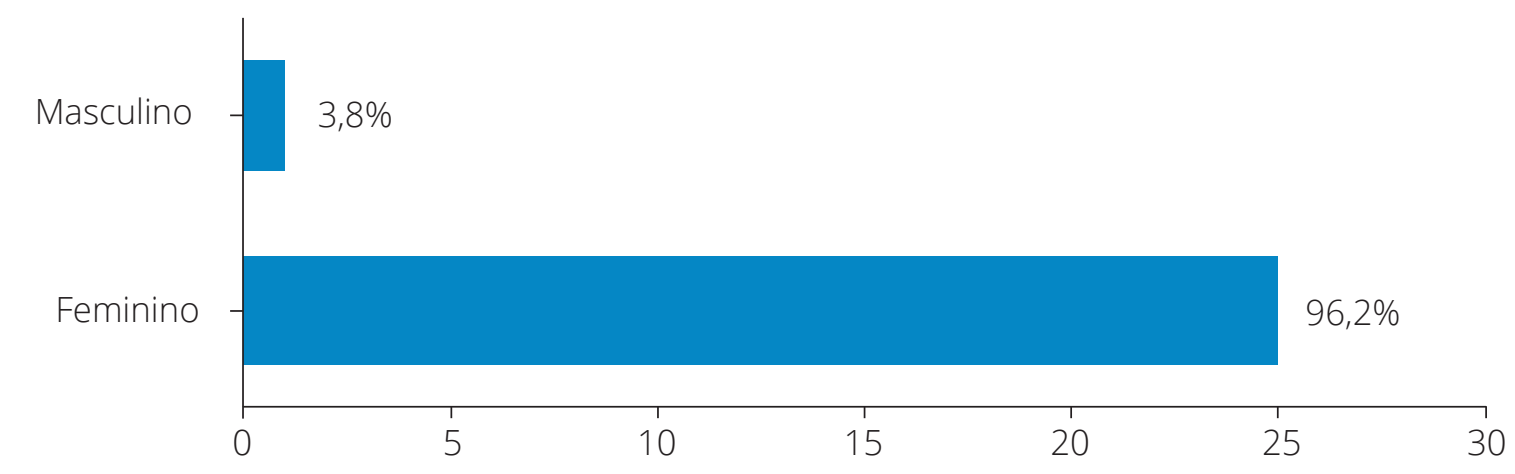

\begin{tabular}{|c|c|c|}
\cline { 2 - 3 } \multicolumn{1}{c|}{} & Feminino & Masculino \\
\hline Sexo & 25 & 1 \\
\hline
\end{tabular}

Gráfico 1. Características sociodemográficas da população do estudo. Salvador, Bahia, 2015.

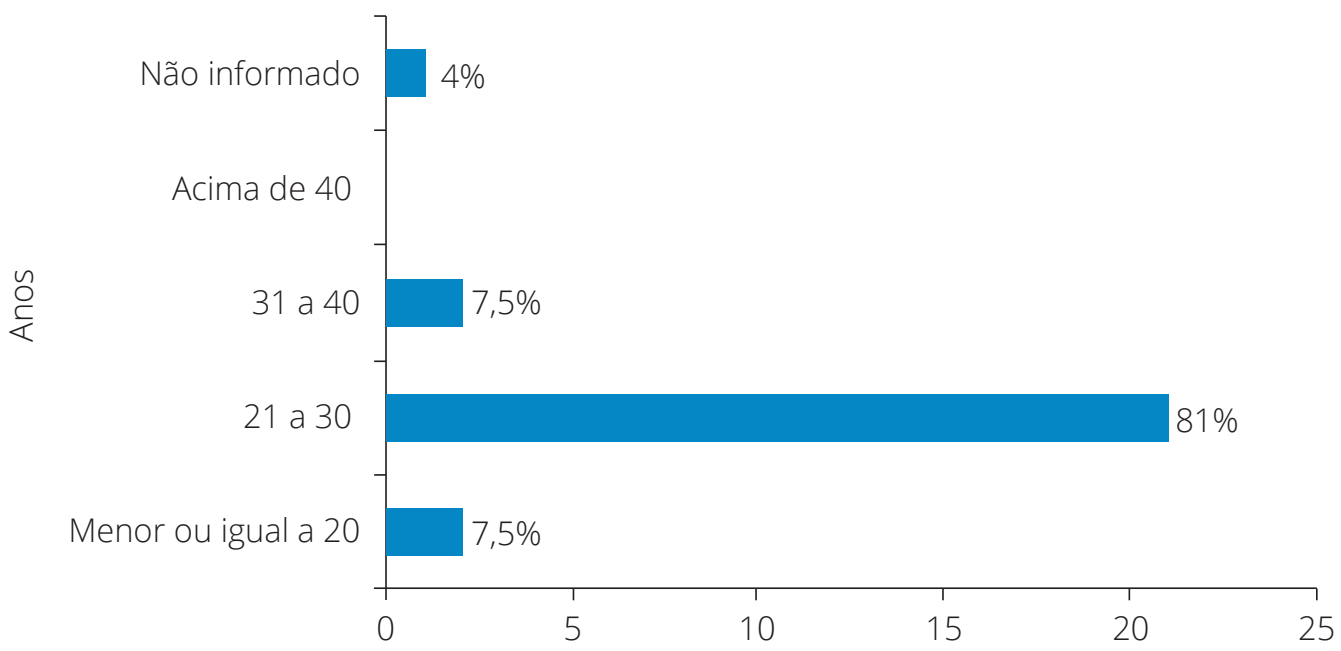

Gráfico 2. Características sociodemográficas da população do estudo. Salvador, Bahia, 2015. 
Tabela 1. Conhecimento dos participantes da pesquisa sobre o monofilamento na cidade de Salvador, Bahia, 2015.

\begin{tabular}{|c|c|c|}
\hline Variável & $\mathrm{n}$ & $\%$ \\
\hline \multicolumn{3}{|c|}{ Conhece o monofilamento de Semmes-Weinstein? } \\
\hline Não & 24 & 92,3 \\
\hline $\operatorname{sim}$ & 2 & 7,7 \\
\hline Total & 26 & 100 \\
\hline \multicolumn{3}{|c|}{ Considera importante a utilização do monofilamento? } \\
\hline Não & 18 & 69,0 \\
\hline $\operatorname{sim}$ & 2 & 8,0 \\
\hline Não informado & 6 & 23,0 \\
\hline Total & 26 & 100 \\
\hline
\end{tabular}

Onde obteve informações sobre monofilamento?

\begin{tabular}{ccc} 
Universidade & 1 & 3,8 \\
\hline Hospital & 0 & 0,0 \\
\hline Unidade básica de saúde & 0 & 0,0 \\
\hline Curso de extensão & 0 & 0,0 \\
\hline Colega de graduação & 2 & 7,7 \\
\hline Não sei o que é monofilamento & 23 & 88,5 \\
\hline Total & 26 & 100
\end{tabular}

Onde adquiriu conhecimento sobre monofilamento?

\begin{tabular}{lcc} 
Internet & 1 & 4,0 \\
\hline Universidade & 0 & 0,0 \\
\hline Curso de extensão & 0 & 0,0 \\
\hline Estágio Curricular & 0 & 0,0 \\
\hline Colega de graduação & 1 & 4,0 \\
\hline Não sei o que é monofilamento & 22 & 84,0 \\
Não informado & 2 & 8,0 \\
Total & 26 & 100
\end{tabular}

Durante o estágio curricular você já presenciou algum profissional utilizando o monofilamento?

\begin{tabular}{lcc} 
Sim & 1 & 4,0 \\
\hline Não & 24 & 92,0 \\
\hline Não informado & 1 & 4,0 \\
\hline Total & 26 & 100 \\
\hline & & Continua...
\end{tabular}

Tabela 1. Continuação.

\begin{tabular}{lcc}
\hline Variável & $\mathbf{n}$ & $\%$ \\
\hline $\begin{array}{l}\text { Sabe informar qual o profissional } \\
\text { habilitado para realização do teste }\end{array}$ & \\
de sensibilidade com monofilamento? & \\
\hline Sim & 2 & 8,0 \\
\hline Não & 22 & 84,0 \\
\hline Não informado & 2 & 8,0 \\
\hline Total & 26 & 100
\end{tabular}

Benefícios da utilização do monofilamento

\begin{tabular}{|c|c|c|}
\hline Diminuir a pressão arterial & 0 & 0,0 \\
\hline Controlar o índice glicêmico & 0 & 0,0 \\
\hline $\begin{array}{l}\text { Reduzir a taxa de ulceração e } \\
\text { amputação }\end{array}$ & 5 & 19,0 \\
\hline Diminuir o índice de colesterol & 0 & 0,0 \\
\hline Controlar dor & 2 & 8,0 \\
\hline Reduzir a taxa lesão & 3 & 11,0 \\
\hline Não informado & 16 & 62,0 \\
\hline Total & 26 & 100 \\
\hline
\end{tabular}

Existe contraindicação para utilização do monofilamento?

\begin{tabular}{ccc} 
Sim & 0 & 0,0 \\
Não & 18 & 69,0 \\
Não informado & 8 & 31,0 \\
Total & 26 & 100 \\
Vantagens do monofilamento de náilon & \\
Armazenamento em baixa & 0 & 0,0 \\
temperatura & 0 & 0,0 \\
Alta durabilidade & 2 & 8,0 \\
Utilização em qualquer paciente & 0 & 0,0 \\
\hline Difícil manuseio & 0 & 0,0 \\
Tempo de execução longo & 23 & 88,0 \\
Não sei & 1 & 4,0 \\
\hline Não informado & 26 & 100 \\
\hline Total & \multicolumn{2}{c}{}
\end{tabular}


náilon, material usado atualmente em todo o mundo, com o mesmo comprimento, mas diâmetros diferentes ${ }^{6,10}$.

Já no Brasil, o kit de monofilamento de náilon começou a ser implantado e utilizado a partir de 1983. A terapeuta ocupacional Linda Lehman foi uma das pioneiras a utilizá-1o ${ }^{11}$.

Esse teste de sensibilidade é de extrema importância, pois irá avaliar o nível de sensibilidade nas regiões em que ele será aplicado, e essa alteração da sensação tátil poderá variar entre normal e perda de sensibilidade tota ${ }^{12}$. Aconselha-se realizá-lo para determinar o risco de ulceração, uma vez que se encontra disponível nas unidades básicas de saúde, é de baixo custo, fácil manuseio, grande sensibilidade e especificidade ${ }^{13}$. Para o paciente, a realização dessa avaliação permite o diagnóstico precoce de neuropatia periférica, reduz o risco de lesão e amputação dos membros e, consequentemente, melhora a sua qualidade de vida.

No entanto, apesar de sua comprovada importância, quando questionados sobre onde obtiveram informações a respeito do monofilamento, $88,5 \%$ dos participantes afirmaram desconhecê-1o, 7,7\% referiram ter obtido informações com os colegas de graduação, e 3,8\%, na universidade. Entre os que o conheciam, 4,0\% adquiram informações na internet, e 4,0\%, nos estágios curriculares da universidade. No entanto, $84,0 \%$ não sabem o que é o monofilamento de Semmes-Weinstein e 8,0\% não informaram. Destaca-se a crescente expansão da internet no meio social e educacional, consolidando-se como recurso para obtenção de informações nas diversas áreas do conhecimento.

Os dados acima mencionados permitem inferir que os discentes da instituição investigada não têm conhecimento suficiente a respeito do monofilamento, possivelmente em decorrência da pouca vivência ou do contato restrito com o tema durante a graduação. Acredita-se também que, embora o teste tenha sido abordado durante o período acadêmico, ainda que de maneira pouco enfática, a busca incansável pelo conhecimento deve partir, sobretudo, do discente, para que possa se tornar um profissional mais completo. Todavia, durante a apresentação do projeto, foi possível notar estranheza por parte dos alunos quando citado o nome científico do teste de sensibilidade.

No que diz respeito à relevância dessa avaliação para as pessoas com diabetes, há de se mencionar que, de acordo com a Sociedade Brasileira de Diabetes, deve ser prestada assistência a esses pacientes em todos os níveis de atenção, e a equipe multidisciplinar deve estar apta e capacitada para oferecê-la com qualidade, bem como orientá-los (inclusive seus familiares) quanto aos cuidados necessários para manutenção de uma boa qualidade de vida, prevenindo, assim, lesões ${ }^{14}$.

Ainda, é responsabilidade do enfermeiro rastrear os pacientes com diabetes, coletar dados importantes sobre sinais e sintomas na anamnese, assim como inspecionar os pés e promover educação continuada, ressaltando a importância do autocuidado e do controle do nível glicêmico baseado em alimentação saudável ${ }^{14,15}$.

Como observado na pesquisa, 92,0\% dos participantes não presenciaram, durante os estágios curriculares, a realização do teste ou não souberam informar o que é o monofilamento, 4,0\% referiram ter visto algum profissional de saúde utilizando o instrumento, e 4,0\% não informaram.

No que se refere ao profissional que realiza o teste, $84 \%$ não souberam informar qual é, 8,0\% relataram no questionário apenas que era aplicado por profissionais de saúde, e 8,0\% não informaram. Esses dados evidenciam que os discentes realmente têm pouca informação e conhecimento sobre o monofilamento de Semmes-Weinstein, o que acarreta significativo déficit. Nota-se, porém, na presente na afirmação que $4,0 \%$ dos participantes da pesquisa referiram ter presenciado a utilização desse teste de sensibilidade.

Importante pontuar que estão habilitados a realizar o teste de monofilamento em paciente com alteração de sensibilidade em região periférica todos os profissionais de saúde que possuem conhecimentos técnicos e científicos para aplicabilidade prática de tal procedimento ${ }^{15}$.

Quando questionados sobre os benefícios da utilização do monofilamento, $62 \%$ não informaram, 19\% afirmaram que reduziria a taxa de ulceração e amputação dos pacientes a ele submetidos, $11 \%$ relataram diminuição de lesões, e $8 \%$ apontaram o controle da dor. $\mathrm{Na}$ verdade, o principal benefício do teste de sensibilidade é auxiliar no diagnóstico precoce de lesões nos nervos, reduzindo, assim, a incidência de lesões. Durante sua aplicação, nos casos em que o paciente não sentir a pressão exercida pelos diferentes diâmetros monofilamentos, ou seja, diante de alteração tátil, ele será orientando pelo profissional de saúde a realizar um acompanhamento médico para tratamento precoce ${ }^{14-16}$.

Os dados desta pesquisa revelaram ainda outro aspecto importante: $69 \%$ dos participantes relataram que, de acordo com o conhecimento por eles obtido, não existe ou eles não souberam informar qual seria a contraindicação para utilização do monofilamento de Semmes-Weinstein; outros 31\% 
não informaram a respeito. Vale, portanto, pontuar que, com base em pesquisas realizadas na Biblioteca Virtual de Saúde (BVS), nos Manuais do Ministério da Saúde e na Scientific Electronic Library Online (SciELO), não há contraindicação para realização do teste de sensibilidade, exceto se houver lesão aparente que dificulte a sua realização, uma vez que, apesar de não ser recomendado utilizar o monofilamento diretamente em região com feridas, pode-se aplicá-lo ao redor de locais lesionados.

Já quando questionados sobre a vantagem na utilização do monofilamento de náilon, $88 \%$ dos participantes não souberam informar, 8,0\% referiram ser aplicável em qualquer paciente, e 4\% não responderam.

Por se tratar de instrumento de avaliação de uso coletivo, recomenda-se, logo após a utilização do monofilamento de náilon, limpá-lo com sabão líquido e água morna, devendo permanecer em repouso durante 24 horas para que possa ser reutilizado. Deve-se ainda evitar contato com agentes químicos. Não há necessidade de esterilização em autoclave, até porque o monofilamento não foi projetado para resistir a altas temperaturas. As vantagens desse instrumento são ser de baixo custo e fácil utilização ${ }^{17}$.

No que se refere à indicação, apenas 7,7\% dos participantes souberam responder. $\mathrm{O}$ exame de monofilamento é indicado para pacientes que tenham alteração de sensibilidade e, consequentemente, possam desenvolver a neuropatia periférica ou outras lesões, como é o caso daqueles com diabetes, hanseníase, infecção por vírus da imunodeficiência humana (HIV), alcoolismo ou em uso de medicações neurotóxicas ${ }^{2}$.

Indagados sobre a técnica utilizada e os locais de aplicação do monofilamento de Semmes-Weinstein, 7,7\% dos participantes souberam responder, embora com pouca precisão. Porém, quanto ao local de aplicação, estes indicaram apenas a região plantar. Já quanto às patologias nas quais é realizado o teste de sensibilidade, referiram somente o diabetes. Nota-se que, apesar de esses dois participantes terem respondido a todas as questões, ainda há déficit de informação quanto à descrição da técnica preconizada. Assim, em razão da ausência de conhecimento suficiente e da larga utilização do teste em programas de diabetes e hanseníase, o tema deveria ser mais bem abordado e discutido em sala de aula.

A respeito da técnica, ainda desconhecida pela maioria dos participantes, deve ocorrer em ambiente tranquilo e confortável para o paciente, com o mínimo de interferência externa ${ }^{16,17}$. Deve-se, primeiramente, colocá-lo na posição sentada, de frente para o examinador, com mãos e pés apoiados e relaxados. É importante realizar uma demonstração prévia e orientá-lo sobre como ocorrerá o exame, fazendo um teste em área de sua pele com sensibilidade normal. Após a demonstração, deve-se ocluir o campo de visão do paciente, utilizando uma barreira. Antes de iniciar o teste, o profissional irá separar os monofilamentos por diâmetro, em ordem crescente, e realizar a aplicação com filamento perpendicular à pele, mantendo-a pressionada por cerca de 1 a 2 segundos e, em seguida, retirando-os suavemente ${ }^{16-19}$.

O profissional irá seguir uma sequência de aplicação, do menor para o maior diâmetro, e a cada aplicação deve perguntar ao paciente se ele sentiu e em que local foi exercida a pressão. Depois disso, deverá anotar, em uma folha própria tal dado, a presença de qualquer alteração ${ }^{18-20}$.

Considera-se diagnóstico suspeito de neuropatia caso o paciente não sinta a pressão exercida pelo monofilamento de $10 \mathrm{~g}$. Nessas situações, torna-se necessário e importante que o enfermeiro enfatize as medidas de prevenção de lesões, além de encaminhar o paciente para acompanhamento médico, a fim de que proceda ao tratamento adequado e evite possíveis consequências ${ }^{15,16}$.

\section{CONCLUSÃO}

A análise dos resultados obtidos permite concluir que a maioria dos discentes participantes não tem conhecimento sobre o monofilamento de Semmes-Weinstein e a sua utilização.

Apesar de o teste estar atualmente disponível nas unidades básicas de saúde e implantado nos programas de saúde a pacientes com diabetes e hanseníase, é ainda pouco conhecido e utilizado por profissionais de saúde, e muito pouco discutido em sala de aula, o que favorece a falta de informação e conhecimento a seu respeito. Visto que os docentes têm um papel importante durante a graduação, seria necessário maior enfoque e divulgação de informação a respeito do teste, para que os discentes tenham maior conhecimento sobre ele.

Diante do exposto, este estudo assume importância no sentido de alertar para essa situação e despertar o desejo dos participantes de buscar conhecimento sobre a utilização desse importante instrumento de saúde. Acredita-se que assim poderão, em futuro próximo, agregar maior valor à futura carreira profissional, visto que estão prestes a concluir o curso de graduação. 
Uma vez que o enfermeiro tem papel importante na rede básica de saúde e pode contribuir para maior aplicação do referido teste de sensibilidade, ele deverá dispor de habilidade e conhecimento para aplicá-lo, assim como para reconhecer as doenças que mais acometem a perda da sensibilidade da região plantar e palmar. Dessa forma, poderá realizar busca ativa desses pacientes e intensificar as atividades de educação continuada dirigidas à valorização do autocuidado, a fim de reduzir o risco de lesão e amputação dos membros e, consequentemente, melhorar a qualidade de vida dessas pessoas.

Sabe-se que a cada ano um número maior de profissionais de Enfermagem se forma e busca o seu espaço no mercado de trabalho, situação que torna premente que os discentes e profissionais já formados busquem por mais conhecimentos e se atualizem constantemente em prol tanto de alcançar sucesso na carreira profissional quanto de melhorar o atendimento àqueles que assistem.

\section{REFERÊNCIAS}

1. Hausen SLJ, Andrew S. Neurologia Clínica de Harrison. $3^{\mathrm{a}}$ ed. Porto Alegre: McGraw-Hill; 2015.

2. Kraychet DC, Sakata RK. Neuropatias periféricas dolorosas. Rev Bras Anestesiol. 2011;61(5):649-58.

3. Sinan. HANSENÍASE Casos confirmados notificados no Sistema de Informação de Agravos de Notificação - Sinan Net [Internet]. 2014 [cited 2015 Jul 14]. Disponível em: http:// www3.saude.ba.gov.br/cgi/tabcgi.exe?tabnet/sinan/hans.def.

4. Ministério da Saúde. DATASUS. Diabetes Tipo 1, Diabetes Tipo 2 segundo Município-Salvador [Internet]. 2012 [cited 2015 Jul 14]. Disponível em: http://tabnet.datasus.gov.br/ cgi/tabcgi.exe?hiperdia/cnv/hdba.def.

5. Smeltzer SC, Bare BG. Brunner \& Suddarth: Tratado de Enfermagem Médico-Cirúrgica. $11^{\mathrm{a}}$ ed. Rio de Janeiro: Guanabara Koogan; 2009.

6. Helene LMF, Leão VM, Minakawa MM. Perfis epidemiológicos e a avaliação de incapacidades físicas de hansenianos de uma UBS de São Paulo. Hansen Int. 2001;26:5-13.

7. Massuia FAO, Uemura GRJRP. Monofilamentos de SemmesWeinstein, Instrumento fundamental ou opcional em mastologia? Ensaios e Ciência: Ciências Biológicas, Agrárias e da Saúde. 2013;17(1):121-8.

8. Brasil. Ministério da Saúde. Guia de controle da hanseníase. $3^{a}$ ed. Brasília: Fundação Nacional da Saúde; 2002.

9. São Paulo. Ribeirão Preto. Secretaria Municipal da Saúde. Protocolo de atendimento em hipertensão e diabetes. Ribeirão Preto: Secretaria Municipal da Saúde; 2011.

10. Moreira D, Escarabel CM. A importância dos monofilamentos de Semmes-Weinstein no exame de sensibilidade do paciente portador de hanseníase. Ciência e Fisioterapia. 2002;1(1):1-5.
11. Moreira D, Alvarez RRA. Utilização dos monofilamentos de Semmes-Weinstein na avaliação de sensibilidade dos membros superiores de pacientes hansenianos atendidos no Distrito Federal. Hansen Int. 1999;24(2):121-8.

12. Brasil. Ministério da Saúde. Secretaria de Estado de Saúde do Distrito Federal. Consenso Internacional sobre Pé Diabético. Grupo de Trabalho Internacional sobre Pé Diabético. Brasília: Ministério da Saúde; 2001.

13. Caiafa JS, Castro AA, Fidelis C, Santos VP, Silva ES, Sitrângulo Junor CJ. Atenção integral ao portador de pé diabético. J VasC Bras. 2011;10(4):1-32.

14. Brasil. Sociedade Brasileira de Diabetes. Tratamento e acompanhamento do diabetes mellitus - diretrizes. Brasília: Sociedade Brasileira de Diabetes; 2009.

15. Santos GILSM, Capirunga JBM, Almeida OSC. Pé Diabético: Condutas do Enfermeiro. Rev Enferm Contemp. 2013;2(1):225-241

16. Brasil. Ministério da Saúde. Fundação Nacional de Saúde. Manual de Prevenção de incapacidades. Brasília: Fundação Nacional de Saúde; 1997.

17. Brasil. Ministério da Saúde. Secretaria de Atenção a Saúde. Caderno de Atenção Básica - Estratégias para o Cuidado da pessoa com Doença Crônica. Brasília: Ministério da Saúde, 2013.

18. Lehman LF, Orsini MBP, Fuzikawa PL, Lima ReC, Gonçalves SD. Avaliação Neurológica Simplificada. Belo Horizonte: ALM International; 1997.

19. González CP. Monofilamento de Semmes-Weinstein. Diabetes práctica. Actualización y habilidades en Atención Primaria. 2010;1(1):8-19.

20. Schmidt MI, Melere C, Damé P. Manual de Procedimento: Monofilamento. Estudo Longitudinal de Saúde do Adulto. ELSA-BRASIL. 2012. [S.I.: s.n.]. 